\title{
The Hilton-Zhao Conjecture is True for Graphs with Maximum Degree 4
}

\author{
Daniel W. Cranston* Landon Rabern ${ }^{\dagger}$
}

May 21, 2019

\begin{abstract}
A simple graph $G$ is overfull if $|E(G)|>\Delta\lfloor|V(G)| / 2\rfloor$. By the pigeonhole principle, every overfull graph $G$ has $\chi^{\prime}(G)>\Delta$. The core of a graph, denoted $G_{\Delta}$, is the subgraph induced by its vertices of degree $\Delta$. Vizing's Adjacency Lemma implies that if $\chi^{\prime}(G)>\Delta$, then $G_{\Delta}$ contains cycles. Hilton and Zhao conjectured that if $G$ is connected with $\Delta \geq 4$ and $G_{\Delta}$ has maximum degree 2 , then $\chi^{\prime}(G)>\Delta$ precisely when $G$ is overfull. We prove this conjecture for the case $\Delta=4$.
\end{abstract}

\section{Introduction and Proof Outline}

A proper edge-coloring of a graph $G$ assigns colors to its edges so that edges receive distinct colors whenever they share an endpoint. The edge-chromatic number of $G$, denoted $\chi^{\prime}(G)$, is the smallest number of colors that allows a proper edge-coloring of $G$. Vizing showed that always $\chi^{\prime}(G) \leq$ $\Delta(G)+1$, where $\Delta(G)$ denotes the maximum degree of $G$. (In this paper, all graphs are simple, which means that every pair of vertices is joined by either 0 or 1 edges.) Since always $\chi^{\prime}(G) \geq \Delta(G)$, we call a graph class 1 when $\chi^{\prime}(G)=\Delta(G)$ and call it class 2 when $\chi^{\prime}(G)=\Delta(G)+1$. For brevity, in what follows we write $\Delta$ to denote $\Delta(G)$, whenever the context is clear.

Erdős and Wilson [4] showed that almost every graph is class 1. In contrast, Holyer [8] showed that it is NP-hard to determine whether a graph is class 1 or class 2. As a result, most work in this area focuses on proving sufficient conditions for a graph to be either class 1 or class 2 . A $k$-vertex is one of degree $k$, and a $k^{-}$-vertex is one of degree at most $k$. A $k$-neighbor (and $k^{-}$-neighbor) of a vertex $v$ is defined analogously. If $G$ is class 1 , then $|E(G)| \leq \Delta\lfloor|V(G)| / 2\rfloor$. This observation motivates the following definition. A graph $G$ is overfull if $|E(G)|>\Delta\lfloor|V(G)| / 2\rfloor$. Every overfull graph is class 2 , since it has more edges than can appear in $\Delta$ color classes. A graph $G$ is critical if $\chi^{\prime}(G)>\Delta$ and $\chi^{\prime}(H) \leq \Delta$ for every proper subgraph $H$. An edge $e \in E(G)$ is a critical edge of $G$ if $\chi^{\prime}(G-e)<\chi^{\prime}(G)$. It is easy to show that every class 2 graph $G$ contains a critical subgraph $H$ with the same maximum degree as $G$. Critical graphs are useful because they have more structure than general graphs. For example, Vizing proved the following.

Vizing's Adjacency Lemma (VAL). Let $G$ be a class 2 graph with maximum degree $\Delta$. If vw is a critical edge of $G$, then $w$ has at least $\max \{\Delta+1-d(v), 2\} \Delta$-neighbors.

\footnotetext{
${ }^{*}$ Department of Mathematics and Applied Mathematics, Viriginia Commonwealth University, Richmond, VA; dcranston@vcu.edu; The first author's research is partially supported by NSA Grant H98230-15-1-0013.

${ }^{\dagger}$ Franklin \& Marshall College, Lancaster, PA; landon.rabern@gmail.com
} 
The core of a graph $G$, denoted $G_{\Delta}$, is the subgraph of $G$ induced by $\Delta$-vertices. VAL shows core that if $G$ is class 2, then $G_{\Delta}$ must contain cycles (this was also proved by Fournier [5]). So a natural question is which class 2 graphs have a core consisting of disjoint cycles. Hilton and Zhao [7] conjectured exactly when this happens. Let $P^{*}$ denote the Petersen graph with a vertex deleted.

Hilton-Zhao Conjecture. If $G$ is a connected graph with $\Delta \geq 3$ and with core of maximum degree at most 2, then $G$ is class 2 if and only if $G$ is $P^{*}$ or $G$ is overfull.

David and Gianfranco Cariolaro [1] proved this conjecture when $\Delta=3$. Kral', Sereni, and Stiebitz [9, p. 57-63] gave an alternate proof. An easy counting argument shows that every graph satisfying the hypotheses of the conjecture has average degree at most $\Delta-1+\frac{\Delta-1}{2 \Delta-3}$; when $\Delta=3$, this is $\frac{8}{3}$. By Lemma 1 below, any counterexample to the conjecture must be critical. Thus, the case $\Delta=3$ is also implied by our result [3] that every critical graph with $\Delta=3$ (other than the Petersen graph with a vertex deleted) has average degree at least $\frac{46}{17} \approx 2.706$.

In this paper, we prove the conjecture when $\Delta=4$. Let $\mathcal{G}_{k}$ denote the class of connected graphs with maximum degree $k$ in which the core has maximum degree at most 2 ; that is, each $k$-vertex has at most two $k$-neighbors. Let $\mathcal{H}_{k}$ denote the class of connected graphs $G$ such that (i) $G$ has maximum degree $k$, (ii) $G$ has minimum degree $k-1$, (iii) $G_{\Delta}$ is a disjoint union of cycles, and (iv) every vertex of $G$ has a $\Delta$-neighbor. Note that $\mathcal{H}_{k} \subseteq \mathcal{G}_{k}$. To prove our main result, we use a lemma of Hilton and Zhao [6], implied by VAL. To keep this paper self-contained, we include a proof.

Lemma 1. If $G \in \mathcal{G}_{k}$ with $k \geq 3$ and $\chi^{\prime}(G)>k$, then $G \in \mathcal{H}_{k}$ and $G$ is critical.

Proof. Let $G$ satisfy the hypotheses and let $H$ be a $k$-critical subgraph of $G$. Suppose $H$ has a $(k-2)^{-}$-vertex $v$. By VAL, $v$ has a $k$-neighbor $w$. Now $w$ has at least $k+1-d(v) \geq k+1-(k-2)=3$ neighbors of degree $k$, a contradiction, since $H \in \mathcal{G}_{k}$. Thus, $H$ has no $(k-2)^{-}$-vertex.

Suppose that $V(H) \subsetneq V(G)$. Choose $v \in V(H)$ and $w \in V(G) \backslash V(H)$ such that $w \in N_{G}(v)$. If $d_{G}(v) \leq k-1$, then $d_{H}(v) \leq k-2$, a contradiction. So $d_{G}(v)=k$. Since $H$ is critical, $v$ has a $k$-neighbor $w$. But now $w$ has at most two $k$-neighbors in $G$ (since $G \in G_{k}$ ), one of which is $v$. So $w$ has at most one $k$-neighbor in $H$, contradicting VAL. Thus, $V(H)=V(G)$. Finally, suppose there exists $e \in E(G) \backslash E(H)$. Now either $H$ has a $(k-2)^{-}$-vertex or some $k$-vertex in $H$ has at most one neighbor $w$ in $H$ with $d_{H}(w)=k$; both are contradictions. Thus, $E(G)=E(H)$. So $G$ is critical. Now VAL implies that every vertex has at least two $\Delta$-neighbors. Hence, $G \in \mathcal{H}_{k}$.

Now we can prove our Main Theorem, subject to three reducibility lemmas, which we state and prove in the next section. In short, the lemmas say that a graph in $\mathcal{H}_{4}$ is class 1 whenever it contains at least one of the configurations in Figure 1 (not necessarily induced).

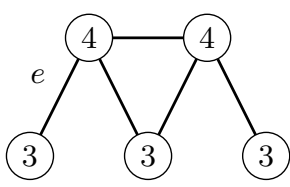

(a)

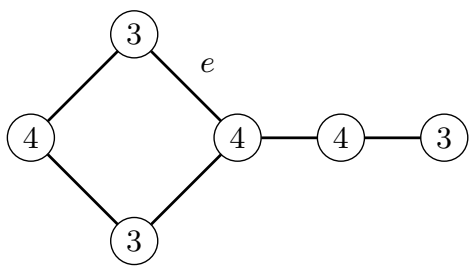

(b)

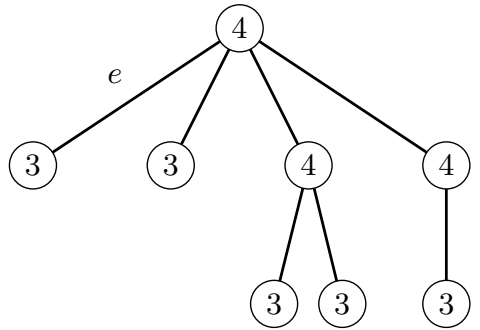

(c)

Figure 1: Each configuration cannot appear in a class 2 graph in $\mathcal{G}_{4}$. (The number at each vertex specifies its degree in $G$.) 
Main Theorem. A connected graph $G$ with $\Delta=4$ and with core of maximum degree at most 2 is class 2 if and only if $G$ is $K_{5}-e$. This implies the case $\Delta=4$ of the Hilton-Zhao Conjecture.

Proof. Let $G$ be a graph with $\Delta=4$ and with core of maximum degree at most 2. By Lemma1, we assume $G \in \mathcal{H}_{4}$. Note that every 4-vertex in $G$ has exactly two 3-neighbors and two 4-neighbors. Let $v$ denote a 4 -vertex and let $w_{1}, \ldots, w_{4}$ denote its neighbors, where $d\left(w_{1}\right)=d\left(w_{2}\right)=3$ and $d\left(w_{3}\right)=d\left(w_{4}\right)=4$. When vertices $x$ and $y$ are adjacent, we write $x \leftrightarrow y$. We assume that $G$ contains no configuration in Figure 1 and show that $G$ is $K_{5}-e$.

First suppose that $v$ has a 3 -neighbor and a 4-neighbor that are adjacent. By symmetry, assume that $w_{2} \leftrightarrow w_{3}$. Since Figure 1(a) is forbidden, we have $w_{3} \leftrightarrow w_{1}$. Now consider $w_{4}$. If $w_{4}$ has a 3-neighbor distinct from $w_{1}$ and $w_{2}$, then we have a copy of Figure 1(b). Hence $w_{4} \leftrightarrow w_{1}$ and $w_{4} \leftrightarrow w_{2}$. If $w_{3} \leftrightarrow w_{4}$, then $G$ is $K_{5}-e$. Suppose not, and let $x$ be a 4-neighbor of $w_{4}$. Since $G$ has no copy of Figure 1(b), $x$ must be adjacent to $w_{1}$ and $w_{2}$. This is a contradiction, since $w_{1}$ and $w_{2}$ are 3 -vertices, but now each has at least four neighbors. Hence, each of $w_{1}$ and $w_{2}$ is non-adjacent to each of $w_{3}$ and $w_{4}$.

Now consider the 3-neighbors of $w_{3}$ and $w_{4}$. If $w_{3}$ and $w_{4}$ have zero or one 3 -neighbors in common, then we have a copy of Figure 1(c). Otherwise they have two 3-neighbors in common, so we have a copy of Figure 1(b).

We first announced the Main Theorem in [2], and included the proof above. But we did not include proofs of the reducibility lemmas that we present in the next section.

\section{Reducibility Lemmas}

In this section we prove the reducibility of the three configurations in Figure 1. More precisely, suppose that $G \in G_{4}$ and $G$ contains one of these configurations, $H$, as a subgraph, not necessarily induced (the number at each vertex of $H$ denotes its degree in $G$ ). We show that $\chi^{\prime}(G)=4$. If not, then Lemma 1 implies that $G \in \mathcal{H}_{4}$ and $G$ is critical. Thus, $\chi^{\prime}(G-e)=4$, where $e$ is the edge denoted in the figure. For convenience, we write coloring to mean edge-coloring with colors $0,1,2,3$. Since $\chi^{\prime}(G-e)=4$, we begin with an arbitrary coloring $\varphi$ of $G-e$. A priori, $\varphi$ could restrict to many possible colorings of $H-e$. Starting from $\varphi$, we use repeated Kempe swaps (see below) to get a coloring of $G-e$ that restricts to one of a few colorings of $H-e$. We conclude by modifying the coloring of $H-e$ to transform the coloring of $G-e$ to a coloring of $G$. At each step, we call the current coloring $\varphi$. So, to change the color of some edge $x y$ to $i$, we "let $\varphi(x y)=i$ ". If $\varphi$ uses color $i$ on an edge incident to vertex $v$, then $v$ sees $i$; otherwise $v$ misses $i$. In the figures that follow, we typically draw all edges incident to vertices of $H$. However, only the edges shown in Figure 1 are considered edges of $H$; the others are pendant edges.

An $(i, j)$-chain at a vertex $v$ is the component containing $v$ of the subgraph induced by edges colored $i$ and $j$. If two vertices $v$ and $w$ are in the same $(i, j)$-chain, then $v$ and $w$ are $(i, j)$-linked; otherwise they are $(i, j)$-unlinked. Each $(i, j)$-chain $P$ is a path or an even cycle. If $P$ is a path that starts in $V(H)$, then $P$ either ends in $V(H)$ or ends in $V(G) \backslash V(H)$. In the latter case, $P$ ends at $\infty$. To recolor an $(i, j)$-chain $P$ means to use color $i$ on each edge colored $j$ and vice versa (this is typically called a Kempe swap, but here we rarely use that term). When $P$ contains a vertex $v$, we also say that we $(i, j)$-swap at $v$. Recoloring any chain in a coloring of $G-e$ yields another coloring of $G-e$. If each $(i, j)$-chain in $G-E(H)$ that starts in $V(H)$ ends at $\infty$, then we can recolor pendant edges independently, by recoloring the chain beginning with each pendant edge. If, instead, an $(i, j)$-chain beginning in $V(H)$ ends in $V(H)$, then its end edges (and endpoints) are paired, and recoloring one edge necessarily recolors the other. Choose $v, w \in V(H)$ that each 
begin an $(i, j)$-chain in $G-E(H)$; call the chains $P_{v}$ and $P_{w}$. If $P_{v}$ and $P_{w}$ both end at $\infty$, the we can simulate that $P_{v}$ ends at $w$, so $P_{v}=P_{w}$. To do so, whenever we recolor $P_{v}$ we also recolor $P_{w}$. Thus, for any pair $(i, j) \subset\{0,1,2,3\}$, we can assume that at most one $(i, j)$-chain in $G-E(H)$ that starts in $V(H)$ ends at $\infty$.

During our process of modifying $\varphi$, when we recolor the $(i, j)$-chain $P$ at $v$, we might want to recolor $P$ but realize that this is no help if $P$ ends at $x$. Similarly, we might also be happy to recolor the $(i, j)$-chain $Q$ at $w$, but realize this also is no help if $Q$ ends at $x$. Fortunately, we can make progress, since it is impossible for both $P$ and $Q$ to end at $x$. To get more control when recoloring, we frequently consider all $(i, j)$-chains in $G-E(H)$ that begin at vertices of $V(H)$. Now our analysis is similar, but more extensive. This approach is possible only when we know the color on every edge of $H-e$. We discuss this general technique further in [2].

In the proofs of the reducibility lemmas, while handling one case, we often reduce to a case handled previously. An alternate approach would be to assume in each case that $G-e$ has no 4-edge-coloring satisfying the hypothesis of any previous case. However, the approach we take has the advantage that it is more easily adapted to give an efficient coloring algorithm.

Lemma 2. Suppose that $\Delta=4$ and $G$ has the configuration in Figure 1(a) (reproduced in Figure 2, if we ignore the colors there). If $\chi^{\prime}(G-v z)=4$, then $\chi^{\prime}(G)=4$.

Proof. We start with a coloring of $G-v z$ and assume that $G$ has no coloring, which leads to a contradiction. We denote by $v^{\prime}, w^{\prime}$, and $x^{\prime}$ the sole unlabeled neighbors of $v, w$, and $x$, respectively. By symmetry, we assume that $z$ sees 0 and 1 , and $v$ sees 0,2 , and 3 . We repeatedly use that $v$ and $z$ must be $(1,2)$ - and (1,3)-linked. We consider three cases: color 0 is used on $v v^{\prime}, v x$, or $v w$.

Case 1: 0 is used on $\boldsymbol{v} \boldsymbol{v}^{\prime}$. Let $\varphi$ be a coloring of $G-v z$, and suppose $\varphi\left(v v^{\prime}\right)=0$. By symmetry, assume that $\varphi(v w)=2$ and $\varphi(v x)=3$, as in Figure 2(a). We show that we may assume all edges are colored as in Figure 2(a). Suppose $\varphi(w x)=0$. Since $v$ and $z$ are (1,3)-linked, $\varphi\left(x x^{\prime}\right)=1$. Now we (1,2)-swap at $x$, which makes $v$ and $z(1,3)$-unlinked, a contradiction. So $\varphi(w x)=1$. Since $v$ and $z$ are $(1,2)$-linked, $\varphi\left(x x^{\prime}\right)=2$.

Suppose $\varphi(w y)=0$, so $\varphi\left(w w^{\prime}\right)=3$. Now $y$ must see 1 ; otherwise a $(0,1)$-swap at $y$ recolors $w x$ with 0 , and $v$ and $z$ become (1,3)-unlinked, a contradiction. So $y$ misses 2 or 3 . Now a $(1,2)$ - or $(1,3)$-swap at $y$ makes $y$ miss 1 , but nothing else has changed. So we are done.

So assume $\varphi(w y)=3$ and $\varphi\left(w w^{\prime}\right)=0$. Now $y$ must see 1 , since $v$ and $z$ are (1,3)-linked. If $y$ misses 2 , then a (1,2)-swap at $y$ makes $y$ miss 1 , a contradiction. So $y$ sees 2 and 1 , and misses 0 . Consider the $(0,1)$-chain $P$ at $y . P$ must contain either (a) $w^{\prime} w, w x$ or (b) $v^{\prime} v$; otherwise we $(0,1)$-swap at $y$ and are done. If (a), then we $(0,1)$-swap at $y$ and are done, since now $v$ and $z$ are

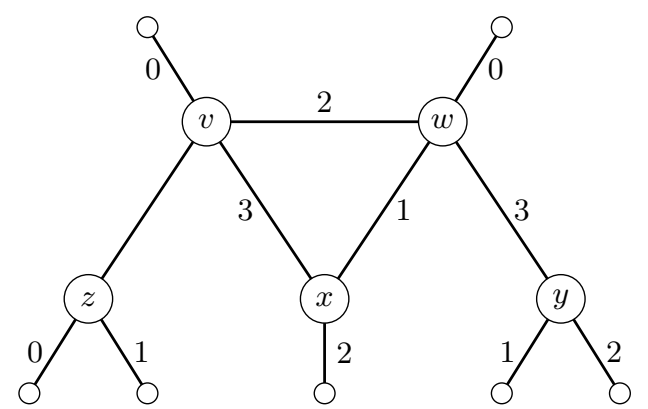

(a)

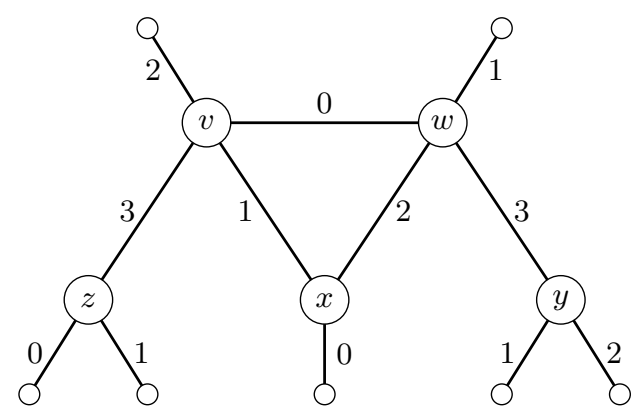

(b)

Figure 2: (a) A coloring of $G-v z$ in Case 1. (b) A coloring of $G$ in Case 2. 
(1,3)-unlinked. So assume (b). Now after a $(0,1)$-swap at $y$, let $\varphi(v x)=0$ and $\varphi(v z)=3$. This completes Case 1.

Case 2: 0 is used on $\boldsymbol{v} \boldsymbol{x}$. The following observation is useful. If no pendant edge uses 3 and edges $v v^{\prime}, w w^{\prime}, x x^{\prime}$ use distinct colors, then $\chi^{\prime}(G)=4$. By symmetry, assume that $\varphi\left(v v^{\prime}\right)=2$, $\varphi\left(w w^{\prime}\right)=1, \varphi\left(x x^{\prime}\right)=0$, as in Figure 2(b). To extend the coloring to $G$, let $\varphi(v z)=3, \varphi(w y)=3$, $\varphi(v w)=0, \varphi(w x)=2, \varphi(v x)=1$.

We now show that we may assume the edges are colored as in Figure 3(a). By symmetry, assume that $\varphi\left(v v^{\prime}\right)=2$ and $\varphi(v w)=3$. Note that $\varphi(w x) \in\{1,2\}$. We assume that $\varphi(w x)=2$. Otherwise $\varphi(w x)=1$ and $\varphi\left(x x^{\prime}\right)=3$, so a $(1,2)$-swap at $x$ gives $\varphi(w x)=2$, as desired. Assume $\varphi\left(x x^{\prime}\right)=3$; otherwise a $(1,3)$-swap at $x$ yields this. Assume $\varphi\left(w w^{\prime}\right)=0$ and $\varphi(w y)=1$; otherwise a $(1,0)$-swap at $w$ yields this. Since $\varphi(v w)=3$ and $\varphi(w y)=1$, vertex $y$ must see 3 . Also, $y$ must see 2 . If not, then we do a $(1,3)$-swap at $x$, followed by a $(1,2)$-swap at $y$, and the resulting $(1,3)$-chain at $v$ ends at $x$. Now do a $(0,1)$-swap at $y$, followed by $(1,3)$-swaps at $x$ and $y$ to ensure that $x$ and $y$ each see 1 . Thus, all edges are colored as in Figure 3(a).

If a $(0,1)$-chain in $G-E(H)$ starts at either $w$ or $x$ and ends at $\infty$, then we are done by the observation at the start of Case 2 . So we assume that the $(0,1)$-chain at $y$ ends at $\infty$, and we recolor it. To maintain a coloring of $G-v z$, let $\varphi(v x)=\varphi(w y)=3$ and $\varphi(v w)=0$, as in Figure 3(b).

Consider the (1,2)-chains in $G-E(H)$ at $v, w, x, y, z$. Let $P$ be the chain at $w$. If $P$ ends at $x$, then we recolor it. To extend the coloring to $G$, let $\varphi(y w)=1, \varphi(w x)=3, \varphi(x v)=1$, and $\varphi(v z)=3$. If $P$ instead ends at $v$, then we again recolor it; now the extension is the same as before, except that $\varphi(v x)=2$. So we must consider three possibilities: the $(1,2)$-chain $P$ at $w$ ends at $y$, ends at $z$, or ends at $\infty$. In each case, we recolor $P$ and show how to get a coloring of $G$.

Suppose $P$ ends at $y$. Recolor it, and let $\varphi(x w)=0$ and $\varphi(w v)=1$, to maintain a coloring of $G-v z$. Now consider the $(0,2)$-chain $Q$ at $w$ in $G-E(H)$. If $Q$ ends at $y$ or $z($ or $\infty)$, then we are done by the observation at the start of Case 2. So assume that $Q$ ends at $v$. Thus, we assume the $(0,2)$-chain at $y$ ends at $z$; now we recolor it and let $\varphi(v z)=0$.

Suppose $P$ ends at $z$. Recolor it, and again let $\varphi(x w)=0$ and $\varphi(w v)=1$. Now consider the $(0,1)$-chains in $G-E(H)$ that start at $x, y$, and $z$; one of them must end at $\infty$. If the chain at $z$ ends at $\infty$, then recolor it and let $\varphi(v z)=0$. If the chain at $x$ ends at $\infty$, then recolor it, and let $\varphi(x w)=1, \varphi(w v)=0$, and $\varphi(v z)=1$. Finally, if the chain at $y$ ends at $\infty$, then recolor it, and let $\varphi(y w)=0, \varphi(w x)=3, \varphi(x v)=0$, and $\varphi(v z)=3$.

So assume $P$ ends at $\infty$. As in the previous case, recolor $P$ and let $\varphi(x w)=0$ and $\varphi(w v)=1$. Now consider the $(0,2)$-chains in $G-E(H)$ that start at $v, w$, and $z$; one such chain must end at $\infty$, so call it $Q$. If $Q$ starts at $v$ or $w$, then we recolor it and are done by the observation at the

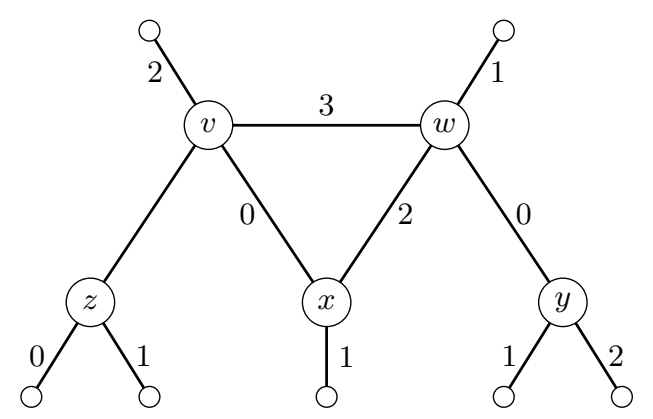

(a)

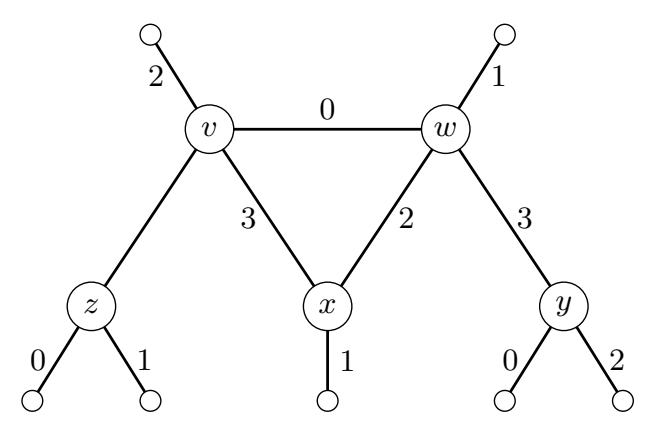

(b)

Figure 3: Two colorings of $G-v z$ in Case 2. 


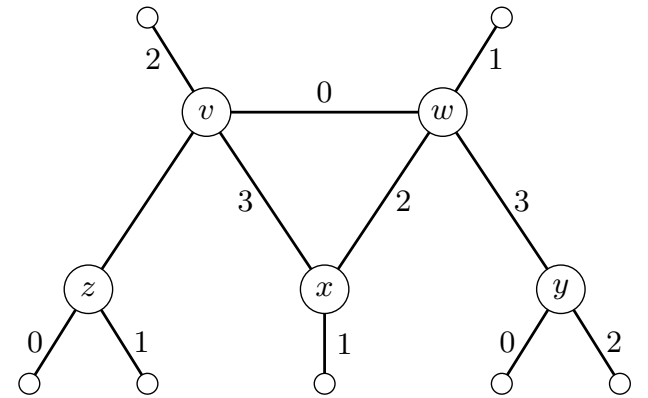

(a)

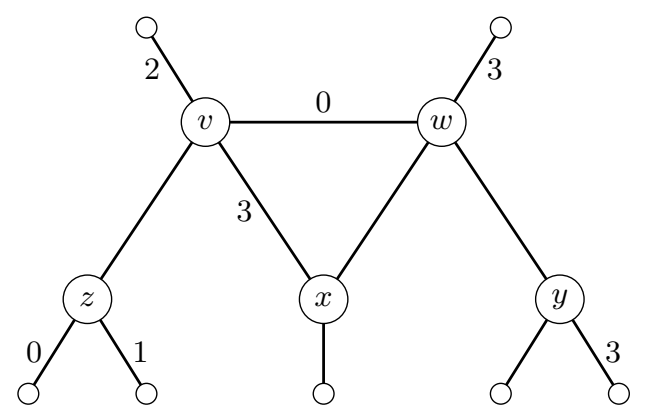

(b)

Figure 4: A coloring and a partial coloring of $G-v z$ in Case 3.

start of Case 2. Otherwise $Q$ starts at $z$, so we recolor $Q$ and let $\varphi(v z)=0$. This completes Case 2 .

Case 3: 0 is used on $\boldsymbol{v} \boldsymbol{w}$. By symmetry, assume that $\varphi\left(v v^{\prime}\right)=2$ and $\varphi(v x)=3$. Note that $x$ must see both 1 and 2; otherwise a (1,2)-swap at $x$ makes $v$ and $z$ (1,3)-unlinked, which is a contradiction. Hence, $x$ misses 0. Suppose that $\varphi(w y)=3$, as in Figure 4(a). Now y must see 0, or else a (0,3)-swap at $x$ reduces to Case 2. We also can assume that $y$ sees 2 and misses 1 , since $v$ and $z$ are $(1,2)$-linked. Now $\varphi(w x) \neq 1$, so $\varphi(w x)=2, \varphi\left(w w^{\prime}\right)=1, \varphi\left(x x^{\prime}\right)=1$. But now we can $(0,1)$-swap at one of $x$ and $y$ without effecting $v w$. Afterwards, either $x$ misses 1 or $y$ misses 0 ; in both cases we are done.

So assume that $\varphi\left(w w^{\prime}\right)=3$, as in Figure 4(b). Suppose that $\varphi(w x)=2$, so $\varphi(w y)=\varphi\left(x x^{\prime}\right)=1$. Now $y$ must see 3 or else a (1,3)-swap at $y$ takes us to the previous paragraph. Note that $v$ and $z$ must be $(0,2)$-linked, or else a $(0,2)$-swap at $x$ reduces to Case 1 . Thus, we can assume that $y$ sees 2 and misses 0. Similarly, $v$ and $z$ must be (0,3)-linked, or we can reduce to Case 2 . However, now a $(0,3)$-swap at $y$ makes $y$ miss 3 , a contradiction. So assume instead that $\varphi(w x)=1$ and $\varphi(w y)=2$. Also $\varphi\left(x x^{\prime}\right)=2$, or else a (1,2)-swap at $x$ makes $v$ and $z(1,3)$-unlinked, a contradiction. Suppose $y$ misses 0 . Now $y$ and $z$ must be $(0,2)$-linked, or else a $(0,2)$-swap at $y$ reduces to Case 2 . But now a $(0,2)$-swap at $x$, followed by a (1,2)-swap at $x$ makes $v$ and $z(1,3)$-unlinked, a contradiction. Thus, $y$ sees 0 . Also, $y$ sees exactly one of 1 and 3 , and we can assume it is 3 . But now a $(1,2)$-swap at $y$ reduces to the case above, where $\varphi(w x)=2$. This completes Case 3 .

Lemma 3. Suppose that $\Delta=4$ and $G$ has the configuration in Figure 1(b) (reproduced in Figure 5 , if we ignore the colors there). If $\chi^{\prime}(G-u x)=4$, then $\chi^{\prime}(G)=4$.

Proof. We start with a coloring of $G-u x$ and assume that $G$ has no coloring, which leads to a contradiction. We denote by $u^{\prime}, w^{\prime}$, and $x^{\prime}$ the sole unlabeled neighbors of $u, w$, and $x$, respectively. By symmetry, we assume that $u$ sees 0 and 1 , and $x$ sees 0,2 , and 3 . We repeatedly use that $u$ and $x$ must be (1,2)- and (1,3)-linked. We consider two cases: color 0 is used on $u u^{\prime}$ or used on $u v$.

Case 1: $\mathbf{0}$ is used on $\boldsymbol{u} \boldsymbol{u}^{\prime}$. We first show that we may assume the edges are colored as in Figure 5. By assumption $\varphi\left(u u^{\prime}\right)=0$ and $\varphi(u v)=1$. We show that $w$ must miss 0. If $\varphi(v w)=0$, then $\varphi(w x)=2$ (by symmetry) and $\varphi\left(w w^{\prime}\right)=1$, but after a (1,3)-swap at $w$, vertices $u$ and $x$ are $(1,2)$-unlinked. A similar argument works if $\varphi(w x)=0$. If $\varphi\left(w w^{\prime}\right)=0$, then $\varphi(w x)=2$ (by symmetry), but now $u$ and $x$ are (1,2)-unlinked, a contradiction. So $w$ misses 0 , as claimed. So, by symmetry, we have $\varphi(v w)=2, \varphi(w x)=3, \varphi\left(w w^{\prime}\right)=1$, as in Figure 5 .

Now we show that $\varphi\left(x x^{\prime}\right)=0$ and $\varphi(x y)=2$. Suppose to the contrary that $\varphi\left(x x^{\prime}\right)=2$ and $\varphi(x y)=0$. Note that $u$ and $w$ must be $(0,2)$-linked; otherwise a $(0,2)$-swap at $w$ gives $\varphi(v w)=0$, a contradiction. If we can get $\varphi(y z)=2$ and $z$ missing 0 , then a $(0,2)$-swap at $z$ gives $\varphi\left(x x^{\prime}\right)=0$ 


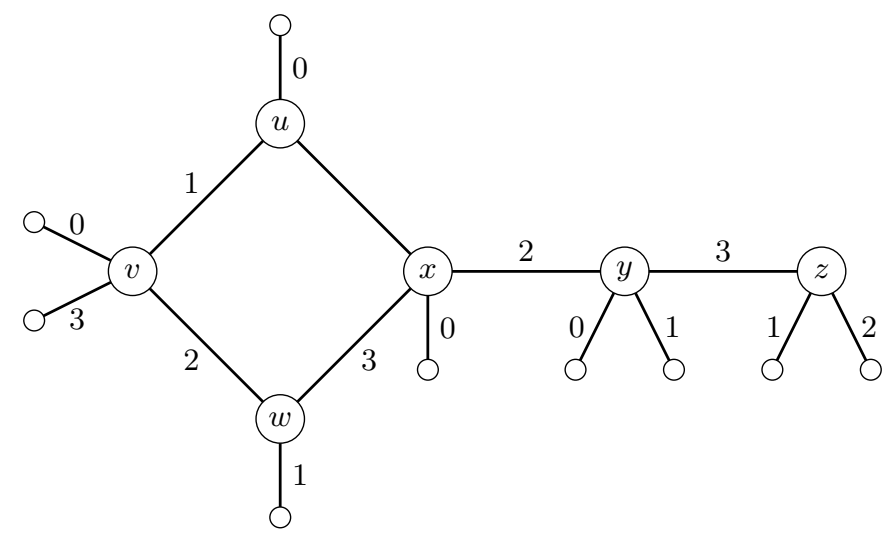

Figure 5: A coloring of $G-u x$ in Case 1 of the proof of Lemma 3 .

and $\varphi(x y)=2$. Always $u$ and $x$ must be (1,2)- and (1,3)-linked. They must also be (0,3)-linked, since otherwise we get a coloring where $w$ sees 0 , a contradiction. Now we use a series of $(0,2)-$, $(0,3)-,(1,2)-$, and $(1,3)$-swaps at $z$ to get $\varphi(y z)=2$ and $z$ missing 0 . (If a $(0,2)$-swap ever recolors $x x^{\prime}$ and $x y$, then we accomplish our goal and are done, so we assume this never happens.) We write $(i ; j)$ to denote that $\varphi(y z)=i$ and $z$ misses $j$. Also $(i ; j) \rightarrow\left(i^{\prime} ; j^{\prime}\right)$ if one of the four swaps mentioned yields $\left(i^{\prime} ; j^{\prime}\right)$ from $(i ; j)$. We have $(3 ; 0) \rightarrow(3 ; 2) \rightarrow(3 ; 1) \rightarrow(1 ; 3) \rightarrow(1 ; 0) \rightarrow(1 ; 2) \rightarrow$ $(2 ; 1) \rightarrow(2 ; 3) \rightarrow(2 ; 0)$. So after a $(2,0)$-swap at $z$, we have $\varphi\left(x x^{\prime}\right)=0$ and $\varphi(x y)=2$, as desired.

Finally, we will show that we may assume $\varphi(y z)=3$ and $z$ misses 0 . In the notation above, we want to reach the case $(3 ; 0)$. We can still use $(0,3)-,(1,2)$-, and $(1,3)$-swaps at $z$ (but, in general, cannot use $(0,2)$-swaps). We have $(0 ; 2) \rightarrow(0 ; 1) \rightarrow(0 ; 3) \rightarrow(3 ; 0)$. We also have $(1 ; 0) \rightarrow$ $(1 ; 3) \rightarrow(3 ; 1) \rightarrow(3 ; 2)$. Further, in $(3 ; 2)$, we can use a $(0,2)$-swap to reach $(3 ; 0)$. For, suppose it interchanges the colors 0 and 2 on $x x^{\prime}$ and $x y$. Now the (0,3)-chain at $z$ ends at $w$. So a (0,3)-swap at $z$ makes $w$ see 0 , a contradiction. Finally, consider $(1 ; 2)$. Now the $(1,2)$-chain at $z$ ends at $x$. After a $(1,2)$-swap at $z$, we let $\varphi(u x)=2$, to get a coloring of $G$. So, we may assume the edges are colored as in Figure 5 .

Let $H$ be the 6-edge-subgraph induced by $\{u, v, w, x, y, z\}$ of the configuration in Figure 5 , Consider the (0,1)-chains in $G-E(H)$ at $u, v, w, x, z$. By parity, one chain must end at $\infty$. Recall that $w$ and $x$ must be $(0,1)$-linked in $G$, since $w$ never sees 0 . So if the $(0,1)$-chain at $w$ or $x$ ends at $\infty$ or $z$, then we reach a contradiction. If the $(0,1)$-chain at $v$ ends at $\infty$ or $z$, then recolor it. Now let $\varphi(v w)=0, \varphi(u v)=2$, and $\varphi(u x)=1$. So assume that the (0,1)-chain at $z$ ends at $\infty$, and recolor it.

Consider the (1,2)-chains in $G-E(H)$ at $w, y$, and $z$. If the $(1,2)$-chain at $w$ ends at $\infty$ or $z$, then recolor it, and let $\varphi(w v)=1, \varphi(v u)=2$, and $\varphi(u x)=1$. So assume the (1,2)-chain at $z$ ends at $\infty$, and recolor it. Finally, consider the (1,3)-chains in $G-E(H)$ that start at $v, w, y$, and $z$. If the chain at $z$ ends at $y$, then recolor it, and let $\varphi(z y)=2, \varphi(y x)=1$, and $\varphi(x u)=2$. If the chain at $z$ ends at $w$, then recolor it and let $\varphi(z y)=2, \varphi(y x)=3, \varphi(x w)=1$, and $\varphi(x u)=2$. If the chain at $y$ ends at $w$, then recolor it and let $\varphi(z y)=2, \varphi(y x)=1, \varphi(x w)=2, \varphi(w v)=1$, $\varphi(v u)=2$, and $\varphi(u x)=3$. This finishes Case 1 .

Case 2: 0 is used on $\boldsymbol{u v}$. We show that we may assume all edges are colored as in Figure 6. After that, a $(0,2)$-swap at $w$ gives $\varphi(w x)=0$, which we handle now. Suppose first that $\varphi(w x)=0$. By possibly using a $(1,2)$ - or $(1,3)$-swap at $w$, we assume that $w$ misses 1 . Now we let $\varphi(w x)=1$, which reduces to Case 1 . So we assume, by symmetry, that $\varphi(w x)=2$. If $w$ sees 0 , then (possibly after a (1,3)-swap at $w$ ), vertex $w$ misses 1 , so $u$ and $x$ are (1,2)-unlinked, a contradiction. Thus, $w$ misses 0. Suppose that $\varphi(v w)=1$ and $\varphi\left(w w^{\prime}\right)=3$. Now we uncolor $w x$ and let $\varphi(u x)=2$. This 


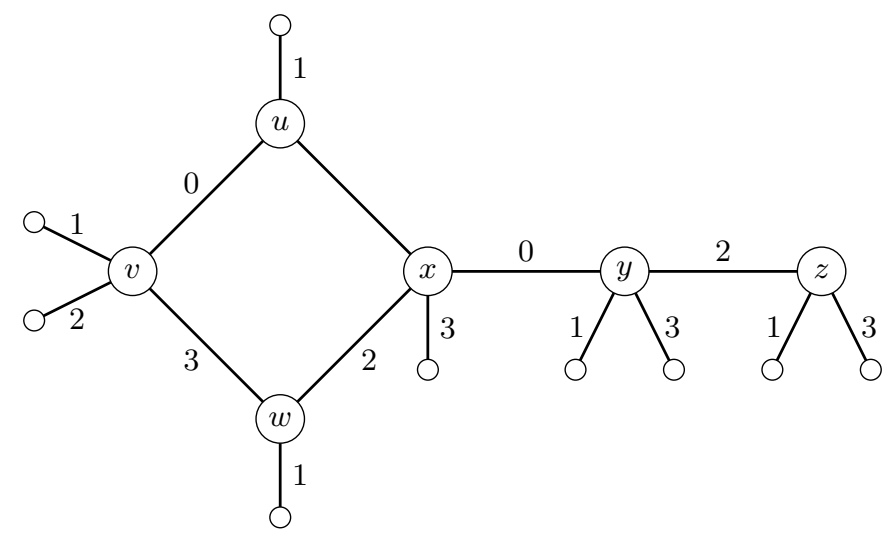

Figure 6: A coloring of $G-u x$ in Case 2 of the proof of Lemma 3 .

reduces to Case 1, with $w$ in place of $u$ (and 3 in place of 0 ). So we assume that $\varphi(v w)=3$ and $\varphi\left(w w^{\prime}\right)=1$, as in Figure 6. Assume that $\varphi\left(x x^{\prime}\right)=3$ and $\varphi(x y)=0$; otherwise, this follows from a $(0,3)$-swap at $x$.

As in Case 1 , we write $(i ; j)$ to denote that $\varphi(y z)=i$ and $z$ misses $j$. Recall that $(1,2)$ - and $(1,3)$-swaps at $z$ do not change the colors on edges incident to $u, v, w$, and $x$. Neither do $(0,2)$ swaps, when $\varphi(y z) \neq 2$. (If $w$ and $u$ are $(0,2)$-unlinked, then we can get $\varphi(w x)=0$, which reduces to Case 1 , as in the previous paragraph.) In fact, we can also use (0,1)-swaps, as follows. Vertices $u$ and $x$ must be $(0,1)$-linked, or we reduce to Case 1 . And $w$ and $x$ must be $(0,1)$-linked, or else we get $w$ missing 1 , which is a contradiction.

We write $(i ; j) \rightarrow\left(i^{\prime} ; j^{\prime}\right)$ if, starting from $(i ; j)$, we get $\left(i^{\prime} ; j^{\prime}\right)$ by using a $(0,1)-,(0,2)-,(1,2)-$, or $(1,3)$-swap at $z$. Our goal is to reach $(2 ; 0)$, as in Figure 6. When we do, the (0,2)-chain at $z$ ends at $w$. Now a $(0,2)$-swap at $z$ gives $\varphi(w x)=0$, which we handled in the first paragraph. Note that $(1 ; 0) \rightarrow(1 ; 2) \rightarrow(2 ; 1) \rightarrow(2 ; 0)$. Also, $(2 ; 3) \rightarrow(2 ; 1) \rightarrow(2 ; 0)$. In any of these five cases, we are done. Note also that $(3 ; 2) \rightarrow(3 ; 0) \rightarrow(3 ; 1) \rightarrow(1 ; 3)$, so we can assume $(1 ; 3)$. Now we use $(0,3)$-swaps at $x$ and $z$. So we have $(1 ; 0)$ and $\varphi\left(x x^{\prime}\right)=0$ and $\varphi(x y)=3$. We use a $(0,2)$-swap at $z$, followed by a $(0,3)$-swap at $x$, followed by a $(1,2)$-swap at $z$, followed by a $(0,1)$-swap at $z$. Now all edges are colored as in Figure 6, so we are done.

Lemma 4. Suppose that $G \in \mathcal{H}_{4}$ and $G$ has the configuration in Figure 1(c) (reproduced in Figure 7 , if we ignore the colors there). If $\chi^{\prime}(G-s t)=4$, then $\chi^{\prime}(G)=4$.

Proof. We start with a coloring of $G-s t$ and assume that $G$ has no coloring, which leads to a contradiction. We denote by $v^{\prime}$ the unlabeled neighbor of $v$. By symmetry, we assume that $t$ sees 0 and 1 , and $s$ sees 0,2 , and 3 . We consider two cases: either 0 is used on $s u$ or it is not.

Case 1: 0 is used on $\boldsymbol{s u}$. By symmetry, assume that $\varphi(s u)=0, \varphi(s v)=2, \varphi(s w)=3$. We will either reach the coloring in Figure 7 (which we show how to extend to st at the end of this case) or else reduce to Case 2: $\varphi(s u) \neq 0$. Since $s$ and $t$ are (1,2)- and (1,3)-linked, we use $(1,2)$ and (1,3)-swaps at $u$ to get $u$ missing 2 (without changing colors on edges incident to $s$ and $t$ ).

We show that we may assume $\varphi(v x)=0$. Suppose not; by symmetry between $x$ and $y$, assume that $\varphi(v x)=3, \varphi(v y)=1$, and $\varphi\left(v v^{\prime}\right)=0$. Now $y$ sees 2 , since $s$ and $t$ are (1,2)-linked. And $u$ must be (0,2)-linked to $t$ (possibly through $w$ and $z$ ) or else we (0,2)-swap at $u$ and reduce to Case 2. If $y$ misses 0 , then a (0,2)-swap at $y$ makes $y$ miss 2 (and thus $s$ and $t$ are (1,2)-unlinked). So assume $y$ sees 0 . After a $(1,3)$-swap at $y$, we have $\varphi(v y)=3$ and $\varphi(v x)=1$, with nothing else changed. So, by the argument above, $x$ sees 0 and 2 . But now the $(1,3)$-chain at $x$ ends at $y$. So 


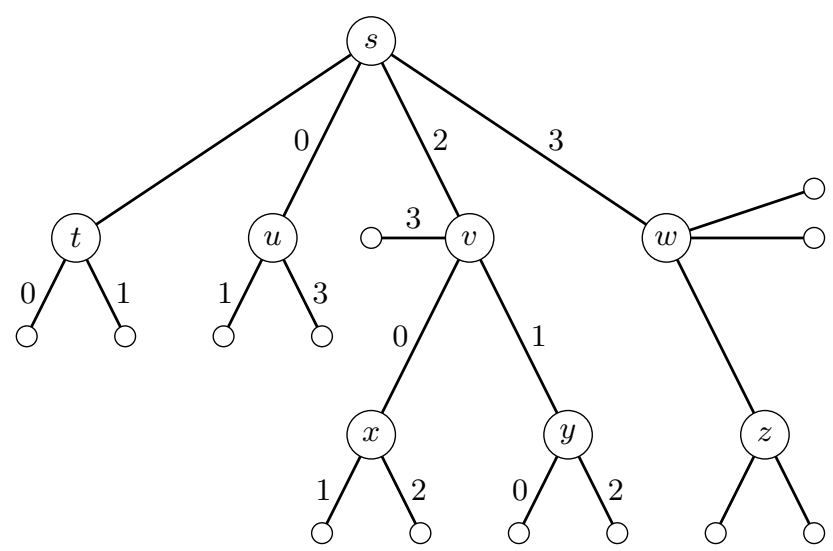

Figure 7: A partial coloring of $G-s t$ in Case 1 of the proof of Lemma 4

either $s$ and $t$ are currently (1,2)-unlinked, or else they become so after a (1,3)-swap at $x$. Thus, we conclude that $\varphi(v x)=0$.

Now we show that we may assume $\varphi(v y)=1$. Assume instead that $\varphi(v y)=3$ and $\varphi\left(v v^{\prime}\right)=1$. Now $x$ sees 2 , or else a $(0,2)$-swap at $x$ reduces to Case 2. If necessary, use a (1,3)-swap at $x$ to get $x$ missing 3 . Note that $y$ sees 1 , or else a $(1,3)$-swap at $y$ gives $\varphi(v y)=1$. If necessary, use a $(0,2)$-swap at $y$ to get $y$ missing 0 . Now the $(0,3)$-chain at $x$ ends at $y$. So either $u$ and $t$ are $(0,2)$-unlinked, or else they become so after a $(0,3)$-swap at $x$. In either case, we use a $(0,2)$-swap at $u$ to reduce to Case 2 . So we must have $\varphi(v x)=0, \varphi(v y)=1, \varphi\left(v v^{\prime}\right)=3$.

Since $s$ and $t$ are (0,2)- and (1,2)-linked, both $x$ and $y$ see 2 . Further, if $y$ misses 0 , then after a $(0,2)$-swap at $y$ vertices $s$ and $t$ are (1,2)-unlinked. Thus, $y$ sees 0 and 2 , and misses 3 . Suppose $x$ misses 1 . Now the (1,2)-chain $P$ at $x$ must end at $u$ (possibly via $w$ and $z$ ); otherwise we recolor $P$, which makes $u$ and $t(0,2)$-unlinked, a contradiction. Now recolor $P$ and let $\varphi(u s)=1, \varphi(s v)=0$, $\varphi(v x)=2, \varphi(s t)=2$. So instead $x$ sees 1 and misses 3, as in Figure 7. Now recolor the (1,3)-chains at $x$ and $y$ (possibly the same chain). Again, the (1,2)-chain $Q$ at $x$ must end at $u$ (possibly via $w$ and $z$ ), since otherwise we recolor it and $u$ and $t$ are $(0,2)$-unlinked. Now Recolor $Q$; as before, let $\varphi(u s)=1, \varphi(s v)=0, \varphi(v x)=2, \varphi(s t)=2$. This completes Case 1 .

Case 2: 0 is not used on su. By assumption $s$ sees 0 . We show that we may assume $\varphi(s w)=0$. Suppose instead that $\varphi(s v)=0$. Since $G \in \mathcal{H}_{4}$, vertex $w$ has two 3-neighbors. If $u \in N(w)$ or $t \in N(w)$, then we have an instance of Figure 1(a), since $z \notin\{t, u\}$. So $\chi^{\prime}(G)=4$, by Lemma 2. Thus, we assume $t, u \notin N(w)$. Now we interchange the roles of $v$ and $w$. (Vertices $v$ and $w$ could have a common 3-neighbor, but this is not a problem.) So $\varphi(s w)=0$. By symmetry, assume that $\varphi(s u)=2$ and $\varphi(s v)=3$. Since $s$ and $t$ are (1,2)-linked, $u$ must see 1 . If $u$ misses 3 , then after a (1,3)-swap at $u$, vertices $s$ and $t$ are (1,2)-unlinked. So $u$ must miss 0 . Thus, we have Figure 8(a), except for colors on edges incident to $z$.

We show that we may assume we have either Figure 8 (a) or else Figure 8(b) with y missing 0. Note that $u$ and $s$ are $(0,1)$-linked, since otherwise we (0,1)-swap at $u$ and finish as above. First, we get $z$ missing 0 . If $z$ sees 0 and misses 1 , then we $(0,1)$-swap at $z$. Otherwise, if $z$ sees 0 it misses 2 or 3 , so after a $(1,2)$ - or $(1,3)$-swap, $z$ misses 1 . These swaps at $z$ do not change the colors on edges incident to $s, t$, or $u$, since $s$ and $t$ are (1,2)- and (1,3)-linked and $s$ and $u$ are (0,1)-linked. Thus $z$ misses 0 . Now consider $\varphi(w z)$. If $\varphi(w z)=1$, then the $(0,1)$-chain at $z$ ends at $s$, so we recolor it and let $\varphi(s u)=0$ and $\varphi(s t)=2$. If $\varphi(w z)=3$, then we are in Figure 8 (a). So assume $\varphi(w z)=2$.

Now consider the 3-neighbor $\widehat{z}$ of $w$, other than $z$. As in the first paragraph of Case 2 , we know $\widehat{z} \notin\{t, u\}$. First suppose $\widehat{z} \notin\{x, y\}$. Now we essentially repeat the argument above, with $\widehat{z}$ in 


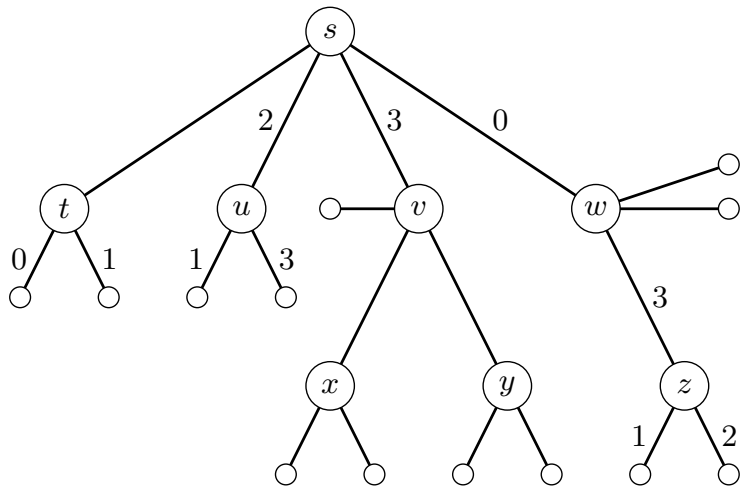

(a)

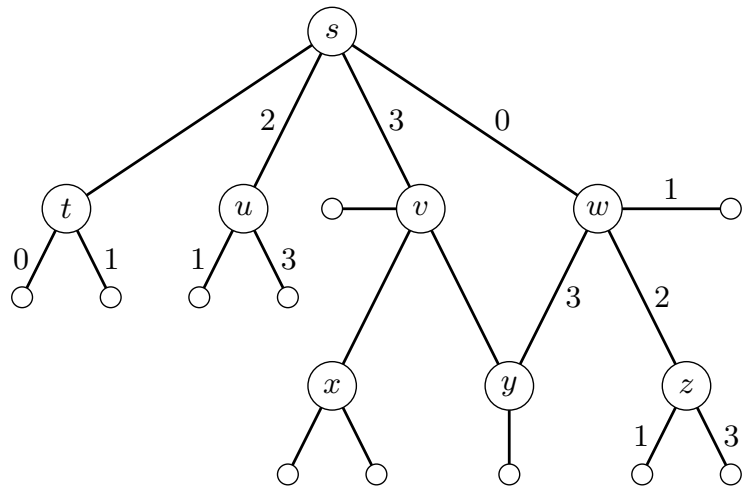

(b)

Figure 8: Two partial colorings of $G-s t$ in Case 2 of Lemma 4 (a) A partial coloring of $G-s t$. (b) A partial coloring of $G-s t$, when $G$ also has edge $w y$.

place of $z$. Suppose $\varphi(w \widehat{z})=3$. If $\widehat{z}$ misses 0 , then we have Figure $[$ (a), with $\widehat{z}$ in place of $z$. If $\widehat{z}$ misses 1 , then a $(0,1)$-swap at $\widehat{z}$ gives that $\widehat{z}$ misses 0 , and we again reach Figure 8 (a); this could make $z$ miss 1 , but that is irrelevant. If $\widehat{z}$ misses 2 , then a (1,2)-swap at $\widehat{z}$ gives that $\widehat{z}$ misses 1 . So instead assume $\varphi(w \widehat{z})=1$. If $\widehat{z}$ misses 0 , then we use a $(0,1)$-swap at $\widehat{z}$, as above. If $\widehat{z}$ misses 2 , then a $(1,2)$-swap at $\widehat{z}$ makes $\varphi(w z)=1$ and $z$ still misses 0 , so we are done. So assume $\widehat{z}$ misses 3 . Now a $(1,3)$-swap at $\widehat{z}$ reduces to the case above where $\varphi(w \widehat{z})=3$. This concludes the case where $\widehat{z} \notin\{x, y\}$. Now suppose $\widehat{z} \in\{x, y\}$; by symmetry, assume that $\widehat{z}=y$. This case is identical, except that we end in Figure 8 (b) with $y$ missing 0 . Thus, we may assume we have either Figure 8 (a) or Figure 8 (b) with $y$ missing 0 . We first consider the latter case, since the argument is simpler.

Case 2a: we have Figure 8(b) with $\boldsymbol{y}$ missing 0. Clearly $\varphi(v x)$ is 2, 1, or 0 . First suppose that $\varphi(v x)=2$, which implies $\varphi(v y)=1$. Now let $\varphi(v y)=3, \varphi(y w)=0, \varphi(w s)=3, \varphi(s v)=1$, $\varphi(s u)=0, \varphi(s t)=2$. So $\varphi(v x) \neq 2$. In what follows, we often use variations on this recoloring idea, typically letting $\varphi(v y)=\varphi(w s)=3$ and $\varphi(w y)=0$, and also recoloring some other edges.

Suppose instead that $\varphi(v x)=1$, which implies $\varphi(v y)=2$; recall that $y$ misses 0 . Now $x$ must see 3 , so $x$ misses 2 or 0 . If $x$ misses 2 , then let $\varphi(v x)=2, \varphi(v y)=3, \varphi(y w)=0, \varphi(w s)=3$, $\varphi(s v)=1, \varphi(s u)=0$, and $\varphi(s t)=2$. So assume $x$ sees 2 and misses 0. Consider the (0,2)-chains at $t, v$, and $x$, and let $P$ be the chain that ends at $\infty$. If $P$ starts at $x$, then recoloring $P$ reduces to the previous case, where $x$ misses 2 . If $P$ starts at $v$, then we recolor $P$ and use nearly the same coloring as above; the only difference is that we let $\varphi(v x)=0$ (rather than $\varphi(v x)=2$ ). So we assume that $P$ starts at $t$, and recolor it.

Now consider the $(0,1)$-chains in $G-E(H)$ at $t, u, v, w, y$, and $z$. If the chain at $t$ ends at $u$, $v, y$, or $z$, then we recolor it (and let $\varphi(v x)=0$ if it ends at $v$ ) and let $\varphi(s t)=1$. So assume $t$ and $w$ are $(0,1)$-linked. Now consider the $(0,1)$-chain $P$ at $u$. If $P$ ends at $z$, then recolor it and let $\varphi(s u)=1, \varphi(s v)=2, \varphi(v y)=3, \varphi(y w)=0, \varphi(w s)=3, \varphi(s t)=0$. If $P$ ends at $v$, then the only difference is that we also let $\varphi(v x)=0$. If $P$ ends at $y$, then nearly the same idea works. Now we recolor $P$, and let $\varphi(s u)=1, \varphi(s v)=2, \varphi(v y)=3, \varphi(y w)=2, \varphi(w z)=0, \varphi(w s)=3, \varphi(s t)=0$. This finishes the case when $\varphi(v x)=1$.

Finally, assume that $\varphi(v x)=0$; again recall that $y$ misses 0 . If $\varphi(v y)=1$, then $\varphi\left(v v^{\prime}\right)=2$. In this case, let $\varphi(v y)=3, \varphi(y w)=0, \varphi(w s)=3, \varphi(s v)=1, \varphi(s u)=0$, and $\varphi(s t)=2$. So assume instead that $\varphi(v y)=2$ and $\varphi\left(v v^{\prime}\right)=1$. We show that if $x$ does not miss 2 , then we can use a $(1,2)$-swap at $x$ (possibly preceded by a (1,3)-swap at $x$ ) to assume that $x$ misses 2 , as follows. If a $(1,3)$-chain starts at $x$, then recoloring it cannot recolor edges incident to $w$, since this would 
make $s$ and $u$ become (0,1)-unlinked. The same is true for a (1,2)-chain. If the (1,2)-chain $P$ at $v$ in $G-v y$ does not end at $t$ or $u$, then we recolor it and let $\varphi(v y)=3, \varphi(y w)=0, \varphi(w s)=3$, $\varphi(s v)=1, \varphi(s u)=0$, and $\varphi(s t)=2$. So $P$ must end at $t$ or $u$. Since $t$ and $u$ are (1,2)-linked in $G$, the (1,2)-chain at $y$ in $G-v y$ also ends at $t$ or $u$. Thus, a (1,2)-swap at $x$ does not recolor edges incident to $t, u, v, w, y$, or $z$. Hence, by using a (1,2)- and (1,3)-swap at $x$, we can assume that $x$ misses 2. Consider the (0,1)-chains in $G-E(H)$ at $u, v, w, x, y, z$. Recall that $u$ and $w$ must be $(0,1)$-linked (possibly through $v$ and $x)$ or else we can recolor the $(0,1)$-chain at $u$ and let $\varphi(u s)=1$ and $\varphi(s t)=2$. So clearly neither $u$ nor $w$ is $(0,1)$-linked to either $y$ or $z$. Further, neither $u$ nor $w$ is $(0,1)$-linked to $x$, since we can recolor that $(0,1)$-chain, let $\varphi(v x)=2, \varphi(v y)=0$, and proceed as before. So $u$ and $w$ must be $(0,1)$-linked. Thus, $v$ is $(0,1)$-linked to $x, y$, or $z$. Let $P$ be the $(0,1)$-chain in $G-E(H)$ at $v$. If $P$ ends at $x$ or $z$, then recolor it and let $\varphi(v x)=2, \varphi(v y)=3$, $\varphi(y w)=0, \varphi(w s)=3, \varphi(s v)=1, \varphi(s u)=0$, and $\varphi(s t)=2$. So assume instead that $P$ ends at $y$. Now recolor $P$ and let $\varphi(v x)=2, \varphi(v y)=3, \varphi(y w)=2, \varphi(w z)=0, \varphi(w s)=3, \varphi(s v)=1$, $\varphi(s u)=0$, and $\varphi(s t)=2$. This completes Case 2 a.

Case 2b: we have Figure 8(a). If a (1,3)-swap elsewhere ever recolors $w z$, then we can finish as in the second paragraph of Case 2 , when $\varphi(w z)=1$. So we assume this never happens. We show that we may assume all edges are colored as in Figure 9. After that, the proof is easy, as we show in the final paragraph below. First, we show that $\varphi(v x)=1$. Suppose not. By symmetry between $x$ and $y$, we assume $\varphi(v x)=2, \varphi(v y)=0$, and $\varphi\left(v v^{\prime}\right)=1$. If $x$ misses 1 , then after a (1,2)-swap at $x$, we have $\varphi(v x)=1$, as desired. If $x$ misses 3 and sees 1 , then a (1,3)-swap at $x$ gets $x$ missing 1. So assume $x$ misses 0 . Note that $u$ and $t$ must be (0,3)-linked; otherwise we use a (0,3)-swap at $u$, followed by a $(1,3)$-swap at $u$, which results in $s$ and $t$ being (1,2)-unlinked, a contradiction. So $y$ must see 3 ; otherwise a (0,3)-swap at $x$ gives $x$ missing 3 (since the (0,3)-chain at $x$ does not interact with other edges shown colored 0 or 3 ). Now $y$ also sees either 1 or 2 . We assume $y$ sees 2; otherwise, we use a (1,2)-swap at $y$ (if this recolors $v x$, then we have $\varphi(v x)=1$, as desired; so assume not). The $(0,1)$-chain at $y$ must end at $z$; otherwise, we recolor it, and have $\varphi(v y)=1$, as desired. So we can recolor the $(0,1)$-chain at $x$ without effecting any other edges shown. Now after a (1,2)-swap at $x$, we have $\varphi(v x)=1$, as desired. Since $s$ and $t$ are (1,3)-linked, $x$ also sees 3 .

Now we show that we may assume $\varphi(v y)=2$ and $\varphi\left(v v^{\prime}\right)=0$. Assume to the contrary that $\varphi(v y)=0$ and $\varphi\left(v v^{\prime}\right)=2$. If $x$ misses 0 and $y$ misses 3 , then a $(0,1)$-swap at $x$ makes $s$ and $t$ be $(1,3)$-unlinked, a contradiction. If $x$ misses 0 and $y$ misses 1 , then a (1,3)-swap at $y$ causes $y$ to miss 3 (as in the previous sentence). So the four possibilities for the ordered pair of colors missed at $x$ and $y$ are $(0,2),(2,1),(2,2),(2,3)$. We reduce to the cases $(2,2)$ and $(2,3)$, as follows. In the case $(2,1)$, a $(1,3)$-swap at $y$ yields $(2,3)$, as desired. Suppose we are in the case $(0,2)$, and use a $(1,2)$-swap at $y$. This must recolor the path through $v x$; otherwise we are in the case $(0,1)$, handled above. Now the $(0,1)$-chain at $y$ must end at $z$, or we recolor it. So we can use a $(0,1)$-swap at $x$. Now $(1,2)$-swaps at $x$ and $y$ yield the case $(2,2)$. So it suffices to handle the cases $(2,2)$ and $(2,3)$.

Suppose we are in the case $(2,2)$; that is, both $x$ and $y$ miss 2 . Use $(1,2)$-swaps at $x$ and $y$, followed by (1,3)-swaps at $x$ and $y$. Now at $x$ we use a (0,3)-swap; this cannot recolor edges incident to $t$ or $u$, since $t$ and $u$ must be (0,3)-linked, as in the first paragraph of Case $2 \mathrm{~b}$. Consider the $(0,1)$-chain $P$ at $v$ in $G-v y$. If $P$ ends at $u, x, y$, or $z$, then recolor $P$ and let $\varphi(v y)=3, \varphi(v s)=1$, and $\varphi(s t)=3$. So assume $P$ ends at $w$. Recall that $s$ and $u$ must be $(0,1)$-linked in $G$. Since $P$ ends at $w$, the $(0,1)$-chain $Q$ at $u$ must end at $y$. Recolor $Q$ and let $\varphi(v y)=3, \varphi(v x)=0, \varphi(v s)=2$, $\varphi(s u)=1$, and $\varphi(s t)=3$.

Finally, assume we are in the case $(2,3)$. Consider the $(0,1)$-chains in $G-E(H)$ starting at $u, w, x, y, z$. Let $P$ be the chain starting at $x$. If $P$ does not end at $w$, then recolor $P$, and let $\varphi(v x)=0, \varphi(v y)=3, \varphi(v s)=1$, and $\varphi(s t)=3$. So $P$ must end at $w$. Let $Q$ be the $(0,1)$-chain starting at $u$. If $Q$ ends at $z$ or $\infty$, then recolor $Q$ and let $\varphi(u s)=1$ and $\varphi(s t)=2$. So $Q$ must end 


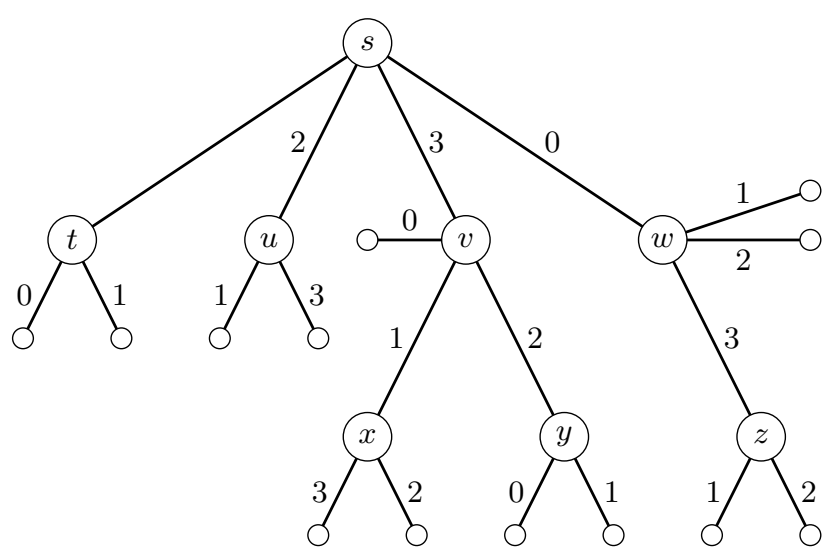

Figure 9: A coloring of $G-s t$ in Case 2 of the proof of Lemma 4

at $y$. Now recolor $Q$, and let $\varphi(y v)=3, \varphi(v s)=0, \varphi(s w)=3, \varphi(w z)=0, \varphi(u s)=1, \varphi(s t)=2$. Thus, we conclude that $\varphi(v y)=2$, and so $\varphi\left(v v^{\prime}\right)=0$.

Now we need only to show that the colors missing at $x$ and $y$ are as in Figure 9. Since $x$ and $t$ are $(1,3)$-linked, $x$ sees 3 , so we have 6 possibilities for these missing colors. If $x$ misses 2 and $y$ misses 3 , then a (1,2)-swap at $x$ makes $s$ and $t(1,3)$-unlinked. If $x$ misses 2 and $y$ misses 1 , then a $(1,3)$-swap at $y$ takes us to the previous sentence. So suppose $x$ misses 2 and $y$ misses 0 . Now a $(1,2)$-swap at $x$ (and interchanging the roles of $x$ and $y$ ) yields the case that $x$ misses 0 and $y$ misses 1. Thus, we assume that $x$ misses 0 . Suppose that $y$ misses 0 . Now $x$ must be $(0,3)$-linked to $v$; otherwise a (0,3)-swap at $x$ makes $s$ and $t$ be (1,3)-unlinked, a contradiction. Now we (0,3)-swap at $y$, after which $y$ misses 3 . This gives the colors in Figure 9. So assume $x$ misses 0 and $y$ misses 1. Now we (1,3)-swap at $y$, which again gives the colors in Figure 9 ,

Finally, we show that if the colors are as in Figure 9, then $G$ has a coloring. Consider the (1,2)-chains in $G-E(H)$ that start at $t, u, x, y$. If the $(1,2)$-chain at $y$ ends at $x$, then recolor it and let $\varphi(v x)=2$ and $\varphi(v y)=1$. Now $s$ and $t$ are (1,3)-unlinked, a contradiction. If the (1,2)-chain at $y$ ends at $u$, then recolor it and let $\varphi(y v)=3, \varphi(v s)=2, \varphi(s u)=1, \varphi(s t)=3$. So assume the $(1,2)$-chain at $y$ ends at $t$. Recolor it and let $\varphi(y v)=3, \varphi(v s)=2, \varphi(s w)=3, \varphi(w z)=0$, $\varphi(s u)=0, \varphi(s t)=1$. This completes Case $2 \mathrm{~b}$, and the proof.

\section{Acknowledgments}

The first author thanks Beth Cranston for encouragement and support during both the research and writing phases of this paper. Thanks also to three anonymous referees; one made suggestions that led to numerous improvements.

\section{References}

[1] D. Cariolaro and G. Cariolaro, Colouring the petals of a graph, Electron. J. Combin., 10 (2003). Paper \#6, pp. 11.

[2] D. W. Cranston And L. RABern, Edge-coloring via fixable subgraphs, https://arxiv.org/abs/1507.05600, (2015).

[3] — Subcubic edge chromatic critical graphs have many edges, J. Graph Theory, 86 (2017), pp. 122-136. Available at: https://arxiv.org/abs/1506.04225

[4] P. ERdős And R. J. Wilson, On the chromatic index of almost all graphs, J. Combin. Theory Ser. B, 23 (1977), pp. 255-257. 
[5] J.-C. Fournier, Méthode et théorème général de coloration des arêtes d'un multigraphe, J. Math. Pures Appl. (9), 56 (1977), pp. 437-453.

[6] A. J. W. Hilton And C. Zhao, The chromatic index of a graph whose core has maximum degree two, Discrete Math., 101 (1992), pp. 135-147. Special volume to mark the centennial of Julius Petersen's "Die Theorie der regulären Graphs", Part II.

[7] - On the edge-colouring of graphs whose core has maximum degree two, J. Combin. Math. Combin. Comput., 21 (1996), pp. 97-108.

[8] I. Holyer, The NP-completeness of edge-coloring, SIAM J. Comput., 10 (1981), pp. 718-720.

[9] M. Stiebitz, D. Scheide, B. Toft, And L. M. Favrholdt, Graph Edge Coloring: Vizing's Theorem and Goldberg's Conjecture, vol. 75, Wiley, 2012. 